\title{
ON THE $K$-THEORY OF ELLIPTIC CURVES
}

\author{
KEVIN P. KNUDSON
}

\begin{abstract}
Let $A$ be the coordinate ring of an affine elliptic curve (over an infinite field $k$ ) of the form $X-\{p\}$, where $X$ is projective and $p$ is a closed point on $X$. Denote by $F$ the function field of $X$. We show that the image of $H_{\bullet}\left(G L_{2}(A), \mathbb{Z}\right)$ in $H_{\bullet}\left(G L_{2}(F), \mathbb{Z}\right)$ coincides with the image of $H_{\bullet}\left(G L_{2}(k), \mathbb{Z}\right)$. As a consequence, we obtain numerous results about the $K$-theory of $A$ and $X$. For example, if $k$ is a number field, we show that $r_{2}\left(K_{2}(A) \otimes \mathbb{Q}\right)=0$, where $r_{m}$ denotes the $m$ th level of the rank filtration.
\end{abstract}

\section{INTRODUCTION}

Computing the $K$-theory of a scheme $X$ is a very difficult task. Even the simplest case $X=\operatorname{Spec} k$, where $k$ is a field, is not completely solved, although a great deal is known. The next case to consider is when $X$ is a curve over $k$, and it is here that the complexity grows rapidly. Some curves of genus zero present no real difficulty thanks to the fundamental theorem: $K_{i}\left(R\left[t, t^{-1}\right]\right)=K_{i}(R) \oplus K_{i-1}(R)$ for $R$ regular. The $K$-theory of elliptic curves, on the other hand, has proved to be much more elusive.

A great deal of recent work has focused on the construction of specific elements in the $K$-theory of elliptic curves, particularly in the second group $K_{2}$. This program goes back to the work of S. Bloch [2], who constructed a regulator map on $K_{2}$ and used it to find nontrivial elements. A. Beilinson [1] generalized this construction and made a number of conjectures relating the dimension of $K_{2} \otimes \mathbb{Q}$ with the values of $L$-functions on the curve. More recently, Goncharov-Levin [5], Rolshausen-Schappacher [9], and Wildeshaus [14] have made further progress in this area.

In this paper we consider the following situation. Let $E$ be an affine elliptic curve defined by the Weierstrass equation $F(x, y)=0$, where

$$
F(x, y)=y^{2}+a_{1} x y+a_{3} y-x^{3}-a_{2} x^{2}-a_{4} x-a_{6} .
$$

Here, the $a_{i}$ lie in an infinite field $k$. Denote by $\bar{E}$ the projective curve $E \cup\{\infty\}$ and by $F$ the function field of $\bar{E}$. Denote by $A$ the affine coordinate ring of $E$; it is a Dedekind domain with field of fractions $F$. We have $A^{\times}=k^{\times}$.

Date: July 13, 1998.

1991 Mathematics Subject Classification. 19D55, 20 G10.

Supported by an NSF Postdoctoral Fellowship, grant no. DMS-9627503. 
Consider the obvious embedding $i: G L_{2}(A) \longrightarrow G L_{2}(F)$. The main result of this paper is the following.

Theorem 1.1. The image of the map

$$
i_{*}: H_{\bullet}\left(G L_{2}(A), \mathbb{Z}\right) \longrightarrow H_{\bullet}\left(G L_{2}(F), \mathbb{Z}\right)
$$

coincides with the image of

$$
\left(\left.i\right|_{G L_{2}(k)}\right)_{*}: H_{\bullet}\left(G L_{2}(k), \mathbb{Z}\right) \longrightarrow H_{\bullet}\left(G L_{2}(F), \mathbb{Z}\right) .
$$

This is a consequence of an explicit computation of the homology of $P G L_{2}(A)$ due to the author [6] (recalled in Section 4 below). The proof of Theorem 1.1 is given in Section 6 .

Remark. Theorem 1.1 and its corollaries in Sections 2 and 3 are valid also for singular cubic curves $F(x, y)=0$. We shall point out the necessary modifications needed to prove this below.

From this result we deduce a number of facts about the $K$-theory of $E$ and $\bar{E}$. Recall the rank filtration of the rational $K$-theory $K_{\bullet}(R)_{\mathbb{Q}}:=K_{\bullet}(R) \otimes_{\mathbb{Z}} \mathbb{Q}$ of a ring $R$ :

$$
r_{m} K_{n}(R)_{\mathbb{Q}}=\operatorname{im}\left\{H_{n}\left(G L_{m}(R), \mathbb{Q}\right) \longrightarrow H_{n}(G L(R), \mathbb{Q})\right\} \cap K_{n}(R)_{\mathbb{Q}} .
$$

Corollary 1.2. The image of the map $r_{2} K_{n}(A)_{\mathbb{Q}} \longrightarrow r_{2} K_{n}(F)_{\mathbb{Q}}$ coincides with the image of $r_{2} K_{n}(k)_{\mathbb{Q}} \longrightarrow r_{2} K_{n}(F)_{\mathbb{Q}}$.

In particular, when $n=2$ we see that the image of $r_{2} K_{2}(A)_{\mathbb{Q}} \longrightarrow$ $r_{2} K_{2}(F)_{\mathbb{Q}}$ coincides with the image of $K_{2}(k)_{\mathbb{Q}}$.

Remark. This corollary is valid for any field $k$. Indeed, if $k$ is finite, then the rational homology $H_{\bullet}\left(G L_{2}(A), \mathbb{Q}\right)$ vanishes in positive degrees (as does $\left.H_{\bullet}\left(G L_{2}(k), \mathbb{Q}\right)\right)$ from which it follows that $r_{2} K_{n}(A)_{\mathbb{Q}}=0$.

Define a filtration $r_{\bullet} K_{\bullet}(\bar{E})_{\mathbb{Q}}$ by pulling back the rank filtration of $K_{\bullet}(A)_{\mathbb{Q}}$ :

$$
r_{m} K_{n}(\bar{E})_{\mathbb{Q}}:=\left(f^{*}\right)^{-1}\left(r_{m} K_{n}(A)_{\mathbb{Q}}\right),
$$

where $f: E \longrightarrow \bar{E}$ is the inclusion and $f^{*}: K_{\bullet}(\bar{E}) \longrightarrow K_{\bullet}(E)=K_{\bullet}(A)$ is the induced map in $K$-theory. Then we obviously have the following result.

Corollary 1.3. The image of $r_{2} K_{n}(\bar{E})_{\mathbb{Q}} \longrightarrow r_{2} K_{n}(F)_{\mathbb{Q}}$ coincides with the image of $r_{2} K_{n}(k)_{\mathbb{Q}} \longrightarrow r_{2} K_{n}(F)_{\mathbb{Q}}$.

We study the filtration $r_{\bullet}$ in greater detail in Section 2. In Section 3 we specialize to the case where $k$ is a number field. In this case, we show that $r_{2} K_{2}(A)_{\mathbb{Q}}=0$.

In the case $n=2$, results of Nesterenko-Suslin [8] imply that $r_{3} K_{2}(A)_{\mathbb{Q}}=$ $K_{2}(A)_{\mathbb{Q}}$. A description of the homology of $P G L_{3}(A)$ (or $G L_{3}(A)$ ) would provide a great deal of insight into the structure of $K_{2}(\bar{E})_{\mathbb{Q}}$, especially over a number field. Such a computation remains elusive, however.

Acknowledgements. I thank Dick Hain for practically insisting that I study this question. Andrei Suslin provided valuable insight regarding the proof of Theorem 1.1. Thanks also go to Mark Walker for answering several (perhaps silly) questions. 


\section{The Rank Filtration}

The rational $K$-groups of affine schemes admit the rank filtration mentioned in the introduction. Since $B G L(R)^{+}$is an $H$-space, the MilnorMoore Theorem [7] implies that the Hurewicz map

$$
h: \pi_{\bullet}\left(B G L(R)^{+}\right) \otimes \mathbb{Q} \longrightarrow H_{\bullet}(G L(R), \mathbb{Q})
$$

is injective with image equal to the primitive elements of the homology. The rank filtration is the increasing filtration defined by

$$
r_{m} K_{n}(R)_{\mathbb{Q}}=\operatorname{im}\left\{H_{n}\left(G L_{m}(R), \mathbb{Q}\right) \longrightarrow H_{n}(G L(R), \mathbb{Q})\right\} \cap K_{n}(R)_{\mathbb{Q}} .
$$

By Theorem 2.7 of $[8]$, the map $H_{2}\left(G L_{3}(A), \mathbb{Z}\right) \rightarrow H_{2}(G L(A), \mathbb{Z})$ is surjective so that $r_{3} K_{2}(A)_{\mathbb{Q}}=K_{2}(A)_{\mathbb{Q}}$. The rank filtration of $K_{2}(A)_{\mathbb{Q}}$ then has the form

$$
0=r_{1} K_{2}(A)_{\mathbb{Q}} \subseteq r_{2} K_{2}(A)_{\mathbb{Q}} \subseteq r_{3} K_{2}(A)_{\mathbb{Q}}=K_{2}(A)_{\mathbb{Q}}
$$

(the vanishing of $r_{1}$ is a consequence of the vanishing of $r_{1} K_{2}(k)_{\mathbb{Q}}$ for infinite fields [8], and the fact that $A^{\times}=k^{\times}$).

Define an increasing filtration $r_{\bullet}$ of $K_{n}(\bar{E})_{\mathbb{Q}}$ as follows. Let $f: E \longrightarrow \bar{E}$ be the canonical inclusion and denote by $f^{*}$ the induced map on $K$-theory. We define $r_{m} K_{n}(\bar{E})_{\mathbb{Q}}$ by

$$
r_{m} K_{n}(\bar{E})_{\mathbb{Q}}=\left(f^{*}\right)^{-1}\left(r_{m} K_{n}(\bar{E})_{\mathbb{Q}}\right) .
$$

There is a commutative diagram

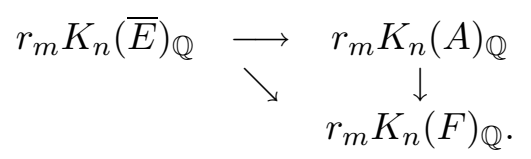

Proposition 2.1. The image of $r_{2} K_{n}(A)_{\mathbb{Q}} \longrightarrow r_{2} K_{n}(F)_{\mathbb{Q}}$ coincides with the image of $r_{2} K_{n}(k)_{\mathbb{Q}} \longrightarrow r_{2} K_{n}(F)_{\mathbb{Q}}$.

Proof. By Theorem 1.1, the image of

$$
i_{*}: H_{\bullet}\left(G L_{2}(A), \mathbb{Z}\right) \longrightarrow H_{\bullet}\left(G L_{2}(F), \mathbb{Z}\right)
$$

coincides with the image of $\left(\left.i\right|_{G L_{2}(k)}\right)_{*}$. Consider the commutative diagram

$$
\begin{array}{ccc}
H_{n}\left(G L_{2}(A), \mathbb{Q}\right) & \longrightarrow & H_{n}(G L(A), \mathbb{Q}) \\
\downarrow & & \downarrow \\
H_{n}\left(G L_{2}(F), \mathbb{Q}\right) & \longrightarrow & H_{n}(G L(F), \mathbb{Q}) .
\end{array}
$$

It follows that the image of $H_{n}\left(G L_{2}(A), \mathbb{Q}\right)$ in $H_{n}(G L(F), \mathbb{Q})$ coincides with the image of $H_{n}\left(G L_{2}(k), \mathbb{Q}\right)$; i.e., the image of $r_{2} K_{n}(A)_{\mathbb{Q}} \rightarrow r_{2} K_{n}(F)_{\mathbb{Q}}$ coincides with the image of $r_{2} K_{n}(k)_{\mathbb{Q}}$.

Corollary 2.2. The image of $r_{2} K_{n}(\bar{E})_{\mathbb{Q}} \longrightarrow r_{2} K_{n}(F)_{\mathbb{Q}}$ coincides with the image of $r_{2} K_{n}(k)_{\mathbb{Q}}$.

Proof. This follows by considering the diagram (1). 


\section{The Number Field Case}

Suppose that the ground field $k$ is a number field. By localizing the projective curve at its generic point we obtain the following exact sequence for $K_{2}$

$$
0 \longrightarrow K_{2}(\bar{E})_{\mathbb{Q}} \longrightarrow K_{2}(F)_{\mathbb{Q}} \stackrel{\mathcal{T}}{\longrightarrow} \bigoplus_{P} K_{1}(k(P))_{\mathbb{Q}}
$$

where $P$ varies over the closed points of $\bar{E}$ and $k(P)$ is the residue field at $P$. The map $\mathcal{T}$ is the tame symbol (see, e.g., [9]).

Remark. It is not known for a single curve if $K_{2}(\bar{E})_{\mathbb{Q}}$ is finite dimensional. Beilinson has conjectured that the dimension of this space is the number of infinite places of $k$ plus the number of primes $\mathfrak{p} \subset \mathcal{O}_{k}$ where $\bar{E}$ has split multiplicative reduction modulo $\mathfrak{p}$. For a discussion of this see, for example, $[9]$.

We also have the localization sequence for $A$ :

$$
\cdots \rightarrow K_{i+1}(F) \rightarrow \bigoplus_{\mathfrak{p} \text { maximal }} K_{i}(A / \mathfrak{p}) \rightarrow K_{i}(A) \rightarrow K_{i}(F) \rightarrow \cdots
$$

Since $A / \mathfrak{p}$ is a finite extension of $k$ for all $\mathfrak{p}$, the groups $K_{2 m}(A / \mathfrak{p})$ are torsion. It follows that we have an exact sequence

$$
0 \longrightarrow K_{2 m}(A)_{\mathbb{Q}} \longrightarrow K_{2 m}(F)_{\mathbb{Q}} \longrightarrow \bigoplus_{\mathfrak{p}} K_{2 m-1}(A / \mathfrak{p})_{\mathbb{Q}}
$$

Proposition 3.1. If the ground field $k$ is a number field, then the map $K_{2}(\bar{E})_{\mathbb{Q}} \rightarrow K_{2}(A)_{\mathbb{Q}}$ is injective.

Proof. This follows by considering the commutative diagram

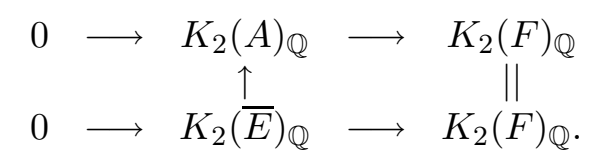

Proposition 3.2. If $k$ is a number field, then $r_{2} K_{2}(A)_{\mathbb{Q}}=0=r_{2} K_{2}(\bar{E})_{\mathbb{Q}}$.

Proof. The map $K_{2}(A)_{\mathbb{Q}} \rightarrow K_{2}(F)_{\mathbb{Q}}$ is injective. But by Proposition 2.1, the image of $r_{2} K_{2}(A)_{\mathbb{Q}}$ coincides with the image of $r_{2} K_{2}(k)_{\mathbb{Q}}=K_{2}(k)_{\mathbb{Q}}=0$.

As a consequence we see that any nontrivial elements of $K_{2}(A)_{\mathbb{Q}}$ (and hence of $\left.K_{2}(\bar{E})_{\mathbb{Q}}\right)$ must come from $H_{2}\left(G L_{3}(A), \mathbb{Q}\right)$. Thus, to prove that $K_{2}(\bar{E})_{\mathbb{Q}}$ is a finite dimensional vector space, it suffices to show that the image of $H_{2}\left(G L_{3}(A), \mathbb{Q}\right)$ in $H_{2}\left(G L_{3}(F), \mathbb{Q}\right)=H_{2}(G L(F), \mathbb{Q})$ is finite dimensional. 


\section{The Homology of $P G L_{2}(A)$}

The remainder of the paper is devoted to the proof of Theorem 1.1. We begin by recalling the calculation of $H_{\bullet}\left(P G L_{2}(A), \mathbb{Z}\right)$ given in $[6]$. The proof uses the action of $P G L_{2}(A)$ on a certain Bruhat-Tits tree $\mathcal{X}$.

We use the description of $\mathcal{X}$ given by Takahashi [13]. Recall that $A$ is the coordinate ring of the affine curve $E$ with function field $F$. The field $F$ has transcendence degree 1 over $k$ and is equipped with the discrete valuation at $\infty, v_{\infty}$. Denote by $\mathcal{O}_{\infty}$ the valuation ring and by $t=x / y$ the uniformizer at $\infty$. Denote by $\mathcal{L}$ the field of Laurent series in $t$ and let $v$ be the valuation on $\mathcal{L}$ defined by $v\left(\sum_{n \geq n_{0}} a_{n} t^{n}\right)=n_{0}$. The ring $A$ can be embedded in $\mathcal{L}$ in such a way that $v(x)=-2$ and $v(y)=-3$; we identify $A$ with its image in $\mathcal{L}$. Note that this embedding induces an embedding $F \rightarrow \mathcal{L}$ and that the completion of $F$ with respect to $v_{\infty}$ is $\mathcal{L}$. We therefore have a commutative diagram

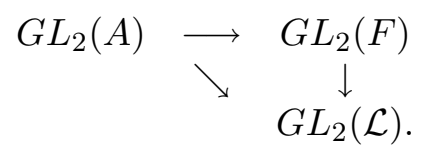

Let $G=G L_{2}(\mathcal{L})$ and $K=G L_{2}(k[[t]])$. Denote by $Z$ the center of $G$. The Bruhat-Tits tree $\mathcal{X}$ is defined as follows. The vertex set of $\mathcal{X}$ is the set of cosets $G / K Z$. Two cosets $g_{1} K Z$ and $g_{2} K Z$ are adjacent if

$$
g_{1}^{-1} g_{2}=\left(\begin{array}{cc}
t & b \\
0 & 1
\end{array}\right) \quad \text { or } \quad\left(\begin{array}{cc}
t^{-1} & 0 \\
0 & 1
\end{array}\right) \quad \text { modulo } K Z
$$

for some $b \in k$. The graph $\mathcal{X}$ is a tree [11], p. 70. Note that $G L_{2}(A)$ acts on $\mathcal{X}$ without inversion and that the center of $G L_{2}(A)$ (which is equal to $\left.k^{\times}\right)$acts trivially on $\mathcal{X}$. It follows that the quotient $P G L_{2}(A) \backslash \mathcal{X}$ is defined. We describe a fundamental domain $\mathcal{D} \subset \mathcal{X}$ for the action (i.e., $\left.\mathcal{D} \cong P G L_{2}(A) \backslash \mathcal{X}\right)$.

If $f_{1}, f_{2} \in \mathcal{L}$, denote by $\phi\left(f_{1}, f_{2}\right)$ the vertex $\left(\begin{array}{cc}f_{1} & f_{2} \\ 0 & 1\end{array}\right) K Z$. Denote by $F_{x}(l, m)$ and $F_{y}(l, m)$ the partial derivatives at $(l, m)$ of the Weierstrass equation $F(x, y)$. Define two sets $E_{1}$ and $E_{2}$ as follows:

$$
E_{1}=\left\{(l, m): F(l, m)=0 \text { and } F_{y}(l, m)=0\right\} \cup\{\infty\}
$$

and

$$
E_{2}=\left\{(l, m): F(l, m)=0 \text { and } F_{y}(l, m) \neq 0\right\} .
$$




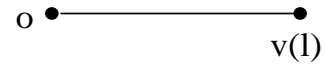

FIGURE 1. $F(l, y)=0$ has no rational solutions

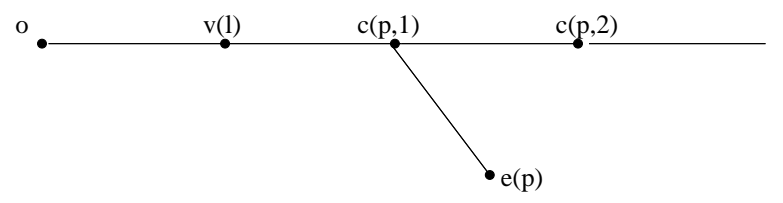

FiguRE 2. $F(l, y)=0$ has a unique rational solution

Observe that $\bar{E}=E_{1} \cup E_{2}$. Define vertices of $\mathcal{X}$ by

$$
\begin{aligned}
o & =\phi\left(t, t^{-1}\right) ; \\
v(l) & = \begin{cases}\phi\left(t^{2}, t^{-1}+l t\right) & \text { if } l \in k \\
\phi\left(1, t^{-1}\right) & \text { if } l=\infty ;\end{cases} \\
c(p, n) & = \begin{cases}\phi\left(t^{n+2}, \frac{y-m}{x-l}\right) & \text { if } p=(l, m) \in E \\
\phi\left(t^{-n}, 0\right) & \text { if } p=\infty ;\end{cases} \\
e(p) & = \begin{cases}\phi\left(t^{4}, \frac{y-m}{x-l}+\frac{F_{x}(l, m)}{y-m}\right) & \text { if } p=(l, m) \in E_{1} \\
\phi(1,0) & \text { if } p=\infty .\end{cases}
\end{aligned}
$$

We are now ready to describe the subgraph $\mathcal{D}$. For each $l \in k \cup\{\infty\}$, the vertex $v(l)$ is adjacent to $o$. Denote by $\mathcal{D}(l)$ the connected component of $\mathcal{D}-\{o\}$ which contains $v(l)$. The $\mathcal{D}(l)$ fall into three types.

(1) Suppose $F(x, y)=0$ has no rational solution with $x=l$. Then $\mathcal{D}(l)$ consists only of $v(l)$ (see Figure 1).

(2) Suppose $l=\infty$ or $F(x, y)=0$ has a unique rational solution with $x=l$. Let $p$ be the point at infinity of $E$ or the rational point corresponding to the solution. Note that $p$ is a point of order 2. Then $\mathcal{D}(l)$ consists of an infinite path $c(p, 1), c(p, 2), \ldots$ and an extra vertex $e(p)$ (see Figure 2).

(3) Suppose $F(x, y)=0$ has two different solutions such that $x=l$. Let $p, q$ be the corresponding points on $E$. Then $\mathcal{D}(l)$ consists of two infinite paths $c(p, 1), c(p, 2), \ldots$ and $c(q, 1), c(q, 2), \ldots$ (see Figure 3$)$.

The infinite path $c(p, 1), c(p, 2), \ldots$ is called a cusp. Note that there is a one-to-one correspondence between cusps and the rational points of $\bar{E}$.

Theorem 4.1 (Takahashi). The graph $\mathcal{D}$ is a fundamental domain for the action of $G L_{2}(A)$ on $\mathcal{X}$ (and hence is also a fundamental domain for the action of $\left.P G L_{2}(A)\right)$.

Remark. The theorem is true also for singular curves $C$ given by $F(x, y)=$ 0 with the following modification. If the curve is singular at $p=(l, m)$, then the vertex $e(p)$ is the same as $c(p, 2)$. In this case, then, the tree $\mathcal{D}(l)$ consists only of the cusp $c(p, 1), c(p, 2), \ldots$ The proofs of the following results for 


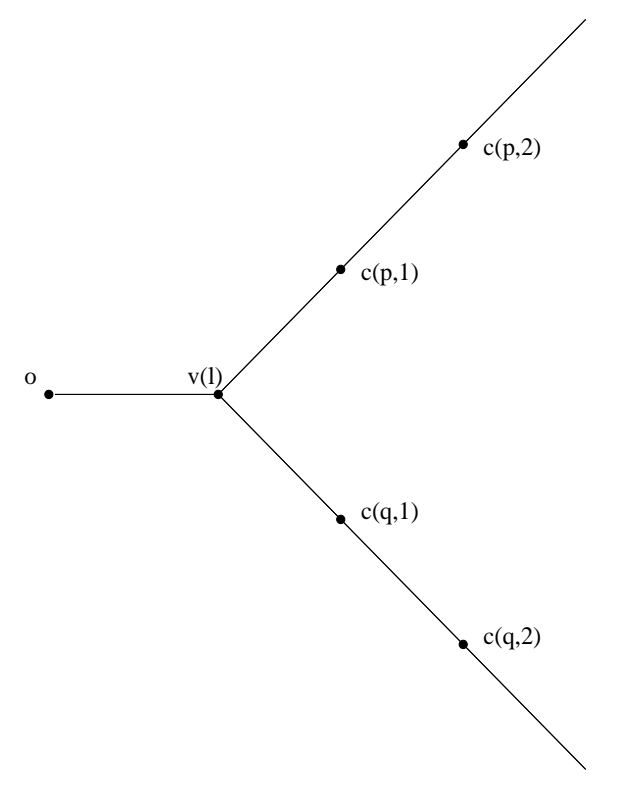

Figure 3. $F(l, y)=0$ has two distinct solutions

$C$ then go through unchanged except that the summands in the homology decomposition of $H_{\bullet}\left(P G L_{2}(k[C]), \mathbb{Z}\right)$ corresponding to singular points are $H_{\bullet}\left(k^{\times}, \mathbb{Z}\right)$ instead of $H_{\bullet}\left(P G L_{2}(k), \mathbb{Z}\right)$.

Since $\mathcal{X}$ is contractible, we have a spectral sequence with $E^{1}$-term

$$
E_{p, q}^{1}=\bigoplus_{\sigma^{(p)} \subset \mathcal{D}} H_{q}\left(\Gamma_{\sigma}, \mathbb{Z}\right) \Longrightarrow H_{p+q}\left(P G L_{2}(A), \mathbb{Z}\right)
$$

where $\Gamma_{\sigma}$ is the stabilizer of the $p$-simplex $\sigma$ in $P G L_{2}(A)$. We shall discuss the stabilizers in detail in the next section. For the purpose of computing homology, the next result is sufficient (see [13], Theorem 5). If $F(l, y)=0$ has no rational solution, denote by $k(\omega)$ the quadratic extension of $k$ in which $F(l, \omega)=0$.

Proposition 4.2. Up to isomorphism, the stabilizers $\Gamma_{\sigma}$ are as follows:

$$
\begin{aligned}
& \Gamma_{o}=\{1\} \\
& \Gamma_{v(l)} \cong \begin{cases}k(\omega)^{\times} / k^{\times} & \text {in case }(1) \\
k & \text { in case }(2) \\
k^{\times} & \text {in case }(3)\end{cases} \\
&\left.\Gamma_{c(p, n)} \cong\left\{\begin{array}{ll}
p & v \\
0 & q
\end{array}\right): p, q \in k^{\times}, v \in k^{n}\right\} / k^{\times} \\
& \Gamma_{e(p)} \cong P G L_{2}(k) .
\end{aligned}
$$

The stabilizer of an edge is the intersection of its vertex stabilizers (one of which is contained in the other). 
By Theorem 1.11 of [8], the inclusion of the diagonal subgroup into $\Gamma_{c(p, n)}$ induces an isomorphism in homology. This leads to the proof of the following, which is the main result of [6].

Theorem 4.3. For all $i \geq 1$,

$$
\begin{aligned}
H_{i}\left(P G L_{2}(A), \mathbb{Z}\right) \cong & \bigoplus_{\substack{l \in k \cup\{\infty\} \\
F(l, y)=0 \text { has unique sol. }}} H_{i}\left(P G L_{2}(k), \mathbb{Z}\right) \\
& \oplus \bigoplus_{\substack{l \in k \\
F(l, y)=0 \text { has two sol. }}} H_{i}\left(k^{\times}, \mathbb{Z}\right) \\
& \bigoplus_{\substack{l \in k \\
F(l, y)=0 \text { has no sol. }}} H_{i}\left(k(\omega)^{\times} / k^{\times}, \mathbb{Z}\right) .
\end{aligned}
$$

Remark. This theorem holds also in degrees $\leq 2$ if $k$ is a finite field with at least 4 elements. For in this case, the inclusion of the diagonal subgroup into $\Gamma_{c(p, n)}$ induces a homology isomorphism in degrees $\leq 2$; see [10], p. 204.

The isomorphism is induced by the inclusion of the various $\Gamma_{v(l)}$ and $\Gamma_{e(p)}$. In the next section, we shall compute the image of the map

$$
H_{\bullet}\left(P G L_{2}(A), \mathbb{Z}\right) \longrightarrow H_{\bullet}\left(P G L_{2}(F), \mathbb{Z}\right) .
$$

\section{The Map $H_{\bullet}\left(P G L_{2}(A), \mathbb{Z}\right) \rightarrow H_{\bullet}\left(P G L_{2}(F), \mathbb{Z}\right)$}

To compute the image of $H_{\bullet}\left(P G L_{2}(A), \mathbb{Z}\right)$ in $H_{\bullet}\left(P G L_{2}(F), \mathbb{Z}\right)$, we must examine the various $\Gamma_{v}$ in greater detail. If $p=\infty$, then the stabilizer $\Gamma_{e(\infty)}$ is the subgroup $P G L_{2}(k)$ of $P G L_{2}(A)$. Hence, under the map $j$ : $P G L_{2}(A) \rightarrow P G L_{2}(F), \Gamma_{e(\infty)}$ maps to $P G L_{2}(k) \subset P G L_{2}(F)$.

The other stabilizers for $l \neq \infty$ are not subgroups of $P G L_{2}(k)$, although they are isomorphic to such. We have the following result.

Theorem 5.1. For each $l \in k$, the stabilizers $\Gamma_{v(l)}$ and $\Gamma_{e(p)}(p=(l, m))$ are conjugate in $P G L_{2}(F)$ to subgroups of $P G L_{2}(k)$.

Corollary 5.2. The image of $j_{*}: H_{\bullet}\left(P G L_{2}(A), \mathbb{Z}\right) \rightarrow H_{\bullet}\left(P G L_{2}(F), \mathbb{Z}\right)$ coincides with the image of $H_{\bullet}\left(P G L_{2}(k), \mathbb{Z}\right)$.

Proof. It is well-known (see [4], p. 48) that conjugation induces the identity on homology. It follows that if $H_{1}, H_{2}$ are conjugate subgroups of a group $G$, then the images of $H_{\bullet}\left(H_{i}, \mathbb{Z}\right) \rightarrow H_{\bullet}(G, \mathbb{Z})$ coincide. Since each stabilizer which appears in the homology decomposition of $P G L_{2}(A)$ is conjugate in $P G L_{2}(F)$ to a subgroup of $P G L_{2}(k)$, the result follows.

Proof of Theorem 5.1. To keep the notation as simple as possible, we only prove the case $\Gamma_{v(0)}$ and in the case $F(0,0)=0=F_{y}(0,0), \Gamma_{e(0,0)}$. All other 
cases are similar (but notationally more complex). For $r_{1}, \ldots, r_{4} \in k$ define

$$
M_{2}\left(r_{1}, r_{2}\right)=\left(\begin{array}{cc}
r_{2} y+r_{1} & -r_{2}\left(\frac{y^{2}+a_{3} y-a_{6}}{x}\right) \\
r_{2} x & -r_{2} y-a_{3} r_{2}+r_{1}
\end{array}\right)
$$

and

$$
\begin{aligned}
& M_{4}\left(r_{1}, r_{2}, r_{3}, r_{4}\right)= \\
& \left.\qquad \begin{array}{cc}
r_{4} x y+r_{3}\left(x^{2}+a_{2} x+a_{4}\right) & -r_{4} y^{2}-r_{3} y\left(x+a_{2}\right)+a_{4} r_{4}\left(x+a_{2}\right) \\
+r_{2} y+r_{1} & -r_{2}\left(x^{2}+a_{2} x+a_{4}-a_{1} y\right) \\
\hline r_{4} x^{2}+r_{3}\left(y+a_{1} x\right) & -r_{4} x y-r_{3}\left(x^{2}+a_{2} x+a_{4}\right) \\
+r_{2} x+a_{4} r_{4} & -r_{2} y+a_{1} a_{4} r_{4}+a_{4} r_{3}+r_{1}
\end{array}\right) .
\end{aligned}
$$

According to Proposition 9 of [13], the stabilizer of $v(0)$ in $G L_{2}(A)$ is

$$
\tilde{\Gamma}_{v(0)}=\left\{M_{2}\left(r_{1}, r_{2}\right): r_{1}\left(-a_{3} r_{2}+r_{1}\right)-a_{6} r_{2}^{2} \neq 0\right\}
$$

and of $e(0,0)$ is

$$
\begin{aligned}
& \tilde{\Gamma}_{e(0,0)}= \\
& \quad\left\{M_{4}\left(r_{i}\right): r_{1}\left(a_{4} r_{3}+r_{1}\right)+\left(-a_{2} a_{4} r_{4}+a_{1} a_{4} r_{3}+a_{4} r_{2} a_{1} r_{1}\right) a_{4} r_{4} \neq 0\right\} .
\end{aligned}
$$

Consider the following identity:

$$
\left(\begin{array}{rr}
x & -y \\
0 & 1
\end{array}\right) M_{2}\left(r_{1}, r_{2}\right)\left(\begin{array}{cc}
1 / x & y / x \\
0 & 1
\end{array}\right)=\left(\begin{array}{cc}
r_{1} & a_{6} r_{2} \\
r_{2} & r_{1}-a_{3} r_{2}
\end{array}\right)=N_{2}\left(r_{1}, r_{2}\right) .
$$

It follows that

$$
\left(\begin{array}{rr}
x & -y \\
0 & 1
\end{array}\right) \tilde{\Gamma}_{v(0)}\left(\begin{array}{cc}
1 / x & y / x \\
0 & 1
\end{array}\right)=\left\{N_{2}\left(r_{1}, r_{2}\right): \operatorname{det} N_{2}\left(r_{1}, r_{2}\right) \neq 0\right\}=\tilde{\Gamma} .
$$

Note that the subgroup $\tilde{\Gamma}$ lies in $G L_{2}(k)$ and that $g=\left(\begin{array}{rr}x & -y \\ 0 & 1\end{array}\right)$ is an element of $G L_{2}(F)$. It follows that $g \Gamma_{v(0)} g^{-1}=\tilde{\Gamma} / k^{\times} \subset P G L_{2}(k)$ inside $P G L_{2}(F)$. Moreover, we can demonstrate the isomorphism of Proposition 4.2 as follows. If $F(0, y)=0$ has no rational solutions, then define a map $\tilde{\Gamma} \rightarrow k(\omega)^{\times}$by $N_{2}\left(r_{1}, r_{2}\right) \mapsto r_{1}+r_{2} \omega$. One checks easily that this is an isomorphism. If $F(0, y)=0$ has two solutions, say $u, v \in k$, then it is easy to see that

$$
\left(\begin{array}{cc}
u & u v \\
\frac{-1}{u(u-v)} & \frac{-1}{u-v}
\end{array}\right) \tilde{\Gamma}\left(\begin{array}{cc}
u & u v \\
\frac{-1}{u(u-v)} & \frac{-1}{u-v}
\end{array}\right)^{-1}=D(k)
$$

where $D(k) \subset G L_{2}(k)$ is the subgroup of diagonal matrices. Finally, if $F(0, y)=0$ has a unique solution, say $u \in k^{\times}$, then

$$
\left(\begin{array}{rr}
0 & -1 \\
1 & u
\end{array}\right) \tilde{\Gamma}\left(\begin{array}{rr}
0 & -1 \\
1 & u
\end{array}\right)^{-1}=B(k)
$$

where $B(k)$ is the upper triangular subgroup of $G L_{2}(k)$. 
For the group $\Gamma_{e(0,0)}$ we have

$$
\left(\begin{array}{cc}
x & -y \\
\frac{y}{a_{4}} & 1-\frac{y^{2}}{a_{4} x}
\end{array}\right) \tilde{\Gamma}_{e(0,0)}\left(\begin{array}{cc}
x & -y \\
\frac{y}{a_{4}} & 1-\frac{y^{2}}{a_{4} x}
\end{array}\right)^{-1}=G L_{2}(k)
$$

from which it follows that $\Gamma_{e(0,0)}$ is conjugate to $P G L_{2}(k)$ inside $P G L_{2}(F)$. (Note that since $\bar{E}$ is smooth, $a_{4} \neq 0$.)

\section{Proof of Theorem 1.1}

We now prove Theorem 1.1. Corollary 5.2 shows that $H_{\bullet}\left(P G L_{2}(A), \mathbb{Z}\right)$ has image equal to the image of $H_{\bullet}\left(P G L_{2}(k), \mathbb{Z}\right)$ in $H_{\bullet}\left(P G L_{2}(F), \mathbb{Z}\right)$. Consider the following commutative diagram

$$
\begin{aligned}
& 1 \rightarrow k^{\times} \rightarrow G L_{2}(A) \rightarrow P G L_{2}(A) \quad \rightarrow \quad 1 \\
& 1 \rightarrow F^{\times} \rightarrow G L_{2}(F) \rightarrow P_{G} L_{2}(F) \rightarrow 1
\end{aligned}
$$

and the induced map of Hochschild-Serre spectral sequences

$$
\begin{array}{ccccc}
E_{p, q}^{2}(A) & = & H_{p}\left(P G L_{2}(A), H_{q}\left(k^{\times}\right)\right) & \Rightarrow & H_{p+q}\left(G L_{2}(A), \mathbb{Z}\right) \\
\downarrow & & \begin{array}{c}
\downarrow \\
\downarrow
\end{array} & & \\
E_{p, q}^{2}(F) & = & H_{p}\left(P G L_{2}(F), H_{q}\left(F^{\times}\right)\right) & \Rightarrow & H_{p+q}\left(G L_{2}(F), \mathbb{Z}\right) .
\end{array}
$$

Since the extensions are central, the groups $H_{q}\left(k^{\times}\right)$(resp. $\left.H_{q}\left(F^{\times}\right)\right)$are trivial $P G L_{2}(A)$ (resp. $P G L_{2}(F)$ ) modules. Hence we have the following commutative diagram of universal coefficient sequences

$$
\begin{array}{cccccc}
H_{p}\left(P G L_{2}(A)\right) \otimes H_{q}\left(k^{\times}\right) & \rightarrow & H_{p}\left(P G L_{2}(A), H_{q}\left(k^{\times}\right)\right) & \rightarrow & \operatorname{Tor}_{1}^{\mathbb{Z}}\left(H_{p-1}\left(P G L_{2}(A)\right), H_{q}\left(k^{\times}\right)\right) \\
\downarrow & & \downarrow & \downarrow & & \\
H_{p}\left(P G L_{2}(F)\right) \otimes H_{q}\left(F^{\times}\right) & \rightarrow & H_{p}\left(P G L_{2}(F), H_{q}\left(F^{\times}\right)\right) & \rightarrow & \operatorname{Tor}_{1}^{\mathbb{Z}}\left(H_{p-1}\left(P G L_{2}(F)\right), H_{q}\left(F^{\times}\right)\right) .
\end{array}
$$

By Corollary 5.2, we see that the image of $E_{p, q}^{2}(A) \rightarrow E_{p, q}^{2}(F)$ coincides with the image of $E_{p, q}^{2}(k) \rightarrow E_{p, q}^{2}(F)$. It follows that the same is true of the $E^{\infty}$ terms:

$$
\operatorname{im}\left\{E_{p, q}^{\infty}(A) \rightarrow E_{p, q}^{\infty}(F)\right\}=\operatorname{im}\left\{E_{p, q}^{\infty}(k) \rightarrow E_{p, q}^{\infty}(F)\right\} .
$$

Thus, the image of $H_{\bullet}\left(G L_{2}(A), \mathbb{Z}\right)$ in $H_{\bullet}\left(G L_{2}(F), \mathbb{Z}\right)$ coincides with the image of $H_{\bullet}\left(G L_{2}(k), \mathbb{Z}\right)$.

\section{REFERENCES}

[1] A. Beilinson, Higher regulators and values of $L$-functions of curves, Funct. Anal. Appl. 14 (1980), 116-118.

[2] S. Bloch, Higher regulators, algebraic $K$-theory, and zeta functions of elliptic curves, Irvine lecture notes, 1977.

[3] A. Borel, J. Yang, The rank conjecture for number fields, Math. Res. Lett. 1 (1994), 689-699.

[4] K. Brown, Cohomology of groups, Springer-Verlag, Berlin/Heidelberg/New York, 1982.

[5] A. Goncharov, A. Levin, Zagier's conjecture on $L(E, 2)$, preprint.

[6] K. Knudson, Integral homology of $P G L_{2}$ over elliptic curves, to appear in Algebraic K-theory, Seattle, WA, 1997, W. Raskind and C. Weibel, eds., Proc. Symp. Pure Math. 
[7] J. Milnor, C. Moore, On the structure of Hopf algebras, Ann. Math. 81 (1965), 211-264.

[8] Yu. Nesterenko, A. Suslin, Homology of the full linear group over a local ring, and Milnor's $K$-theory, Math. USSR Izvestiya 34 (1990), 121-145.

[9] K. Rolshausen, N. Schappacher, On the second $K$-group of an elliptic curve, J. reine angew. Math. 495 (1998), 61-77.

[10] J. Rosenberg, Algebraic $K$-theory and its applications, Springer-Verlag, Berlin/Heidelberg/New York, 1994.

[11] J.-P. Serre, Trees, Springer-Verlag, Berlin/ Heidelberg, New York, 1980.

[12] C. Soulé, Opérations en $K$-théorie algébrique, Canad. J. Math. 37 (1985), 488-550.

[13] $S$. Takahashi, The fundamental domain of the tree of $G L(2)$ over the function field of an elliptic curve, Duke Math. J. 73 (1993), 85-97.

[14] J. Wildeshaus, On an elliptic analogue of Zagier's conjecture, Duke Math. J. 87 (1997), 355-407.

Department of Mathematics, Northwestern University, Evanston, IL 60208

E-mail address: knudson@math.nwu.edu 